\title{
Relaciones entre Nervio Mediano y Musculo Pronador Redon- do en la Región Cubital: Implicancias Anatomo-Clínicas
}

\author{
Relationships between Median Nerve and the Pronator Teres Muscle \\ in the Cubital Region: Anatomic and Clinical Implications
}

\author{
Riveros, A. ${ }^{* * *}$; Olave, E. ${ }^{* * * * * *}$ \& Sousa-Rodrigues, C. $^{* * * *}$
}

RIVEROS, A.; OLAVE, E. \& SOUSA-RODRIGUES, C. Relaciones entre nervio mediano y musculo pronador redondo en la región cubital: implicancias anatomo-clínicas. Int. J. Morphol., 33(4):1448-1454, 2015.

RESUMEN: La intensa actividad muscular del antebrazo genera la necesidad de describir los posibles sitios de compresión de los nervios periféricos del miembro superior. En el caso del nervio mediano, uno de los sitios con mayor prevalencia de atrapamiento es cuando el nervio transita en el interior del canal del carpo. A este sitio debemos sumar la potencial compresión de este nervio en su paso entre las cabezas del músculo pronador redondo (PR). Esta compresión es caracterizada por paresias de músculos flexores de muñeca o algunos músculos de la eminencia tenar, signos a los que se pueden sumar parestesias y dolor en la mano. El objetivo del presente estudio fue describir la relación del nervio mediano con el músculo pronador redondo, para ello se utilizaron 30 antebrazos, de cadáveres formolizados, de individuos brasileños, adultos. Se encontró que en un $40 \%$ de los casos el nervio mediano atravesó la cabeza ulnar del músculo PR a lo que se sumó un caso en la cual la cabeza ulnar del músculo PR estuvo ausente, por lo cual, dicho nervio transitó posterior a la cabeza humeral del citado músculo. La clínica que se desarrolla a partir de un síndrome de compresión a este nivel está directamente vinculado a la distribución de los ramos que el nervio presenta proximal al sitio de la compresión. Asociado a estos hallazgos, resulta vital que para diagnosticar de manera óptima una compresión del nervio mediano es preciso descartar una compresión de este nervio en sitios proximales al canal del carpo.

PALABRAS CLAVE: Anatomía; Nervio mediano; Músculo pronador redondo; Síndrome de compresión.

\section{INTRODUCCIÓN}

La musculatura presente en el antebrazo tiene como principal función permitir una movilidad libre y precisa de la mano y en particular de sus dedos. Si a lo anterior sumamos el excesivo uso de esta importante musculatura, es que se genera la necesidad de describir los posibles sitios de compresión de los nervios periféricos, que en su tránsito hacia las regiones distales del miembro superior pueden sufrir fenómenos de compresión. En el caso del nervio mediano uno de los síndromes de compresión con mayor prevalencia se desarrolla en su tránsito por el interior del canal del carpo, al que debemos sumar sitios proximales como aquel que se relaciona con el paso de este nervio entre las cabezas de origen del músculo pronador redondo (PR), que cuando comprimido se manifiesta con paresias de músculos flexores de la muñeca o debilidad en la oposición del pulgar, a lo que se suman parestesias y dolor en antebrazo y mano (Tubbs et al., 2010).
En este sentido, autores como Rauber (1898), Gérard (1921), Testut \& Latarjet (1972), Nigst \& Dick (1979), Sinelnikov (1986), Gross \& Jones (1992), Williams et al. (1998) \& Rouvière \& Delmas (2005) describieron que cuando el nervio mediano abandona la fosa cubital, lugar en donde toma relación con el musculo PR, atraviesa este músculo entre sus dos cabezas de origen, en cambio para Sappey (1889) y Prives et al. (1984), en este lugar el nervio mediano transita posterior a la cabeza ulnar del PR.

Con respecto a lo anterior, Beaton \& Anson (1939) estudiaron 240 miembros superiores, encontrando que en un $82,5 \%$ de los casos, el nervio mediano se ubicó entre las cabezas de origen del pronador redondo, mientras que en el $8,75 \%$, la cabeza ulnar del pronador redondo estuvo ausente, así mismo, en un 6,25\% el nervio mediano se hizo posterior a las dos cabezas del pronador redondo, dejando

\footnotetext{
* Departamento de Ciencias Morfológicas, Facultad de Ciencias, Universidad San Sebastián, Concepción, Chile.

** Programa de Magíster en Ciencias mención Morfología, Universidad de La Frontera, Temuco, Chile.

**** Facultad de Medicina, Universidad de La Frontera, Temuco, Chile.

${ }^{* * * * *}$ Universidad Estadual de Ciencias da Saude de Alagoas, Maceió, Brasil.
} 
un $2,5 \%$ en la cual el nervio mediano se dispuso a través del vientre muscular de la cabeza humeral de este músculo. Otro antecedente aportado por von Lanz \& Wachsmuth (1959), es que en un 95\% de los casos, el nervio discurre entre las cabezas del músculo PR, dejando al $2 \%$ de estos casos como aquellos en donde el nervio mediano perforó la cabeza humeral del músculo PR y al 3\% faltante como aquel en donde el nervio transitó por debajo de la cabeza ulnar, directamente adherido a la epífisis proximal de la ulna.

Basado en lo descrito, el estudio tuvo por objetivo determinar el trayecto del nervio mediano en la región cubital y su relación con las cabezas del músculo PR, para así evidenciar la posibilidad de compresión de este nervio.

\section{MATERIAL Y METODO}

En este estudio se utilizaron 30 antebrazos, de individuos adultos Brasileños, quienes pertenecen a la Facultad de Medicina de la Universidad Estadual de Ciencias da Saúde de Alagoas, Maceió, Brasil. Estos cadáveres se encontraban fijados con una solución de formaldehído al 10\%. Este estudio se formuló bajo una metodología de carácter cuantitativo y descriptivo, para la cual se procedió a realizar una detallada disección del compartimento anterior del antebrazo con material quirúrgico ad-hoc.

El punto de referencia seleccionado para las mediciones realizadas fue el epicóndilo medial del humero y las mediciones obtenidas fueron realizadas mediante la utilización de un caliper digital marca Mitutoyo de 0,01 $\mathrm{mm}$ de precisión.

\section{RESULTADOS}

En su ingreso a la fosa cubital el nervio mediano presentó un diámetro promedio de $4,8 \pm 1,04 \mathrm{~mm}$, medición que se realizó a la altura de la linea biepicondilar (LBE). Respecto a este punto de referencia el nervio mediano recorrió una distancia promedio 53,5 $\pm 11,91 \mathrm{~mm}$, hasta alcanzar las cabezas de origen del músculo pronador redondo.

En relación al detalle del trayecto que el nervio tomó respecto a las cabezas de origen del múscu-

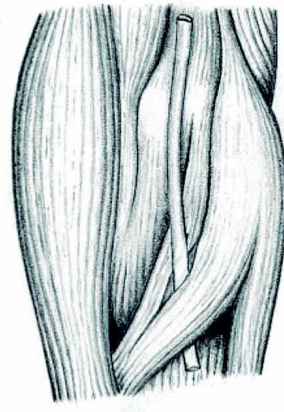

$57 \%$

Entre las cabezas del $\mathrm{m}$. pronador redondo

Fig. 1. Esquema que muestra el trayecto del nervio mediano en relación al músculo pronador redondo.

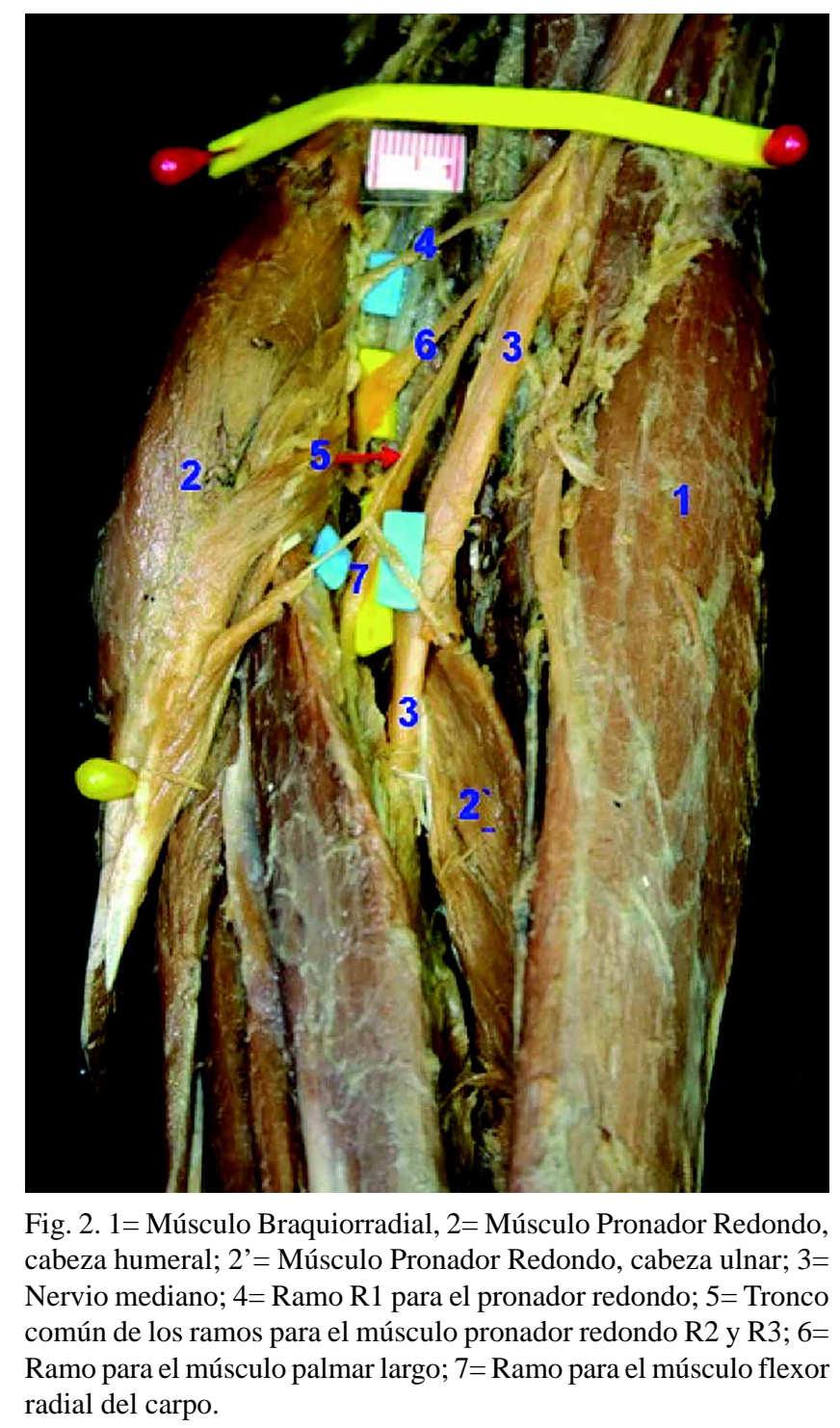

Fig. 2. 1= Músculo Braquiorradial, 2= Músculo Pronador Redondo, cabeza humeral; 2' = Músculo Pronador Redondo, cabeza ulnar; 3= Nervio mediano; 4= Ramo R1 para el pronador redondo; 5= Tronco común de los ramos para el músculo pronador redondo R2 y R3; 6= Ramo para el músculo palmar largo; 7= Ramo para el músculo flexor radial del carpo.

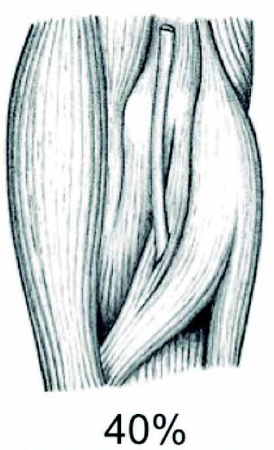

Perforó cabeza ulnar del $\mathrm{m}$. pronador redondo

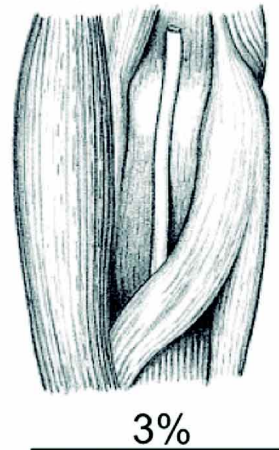

Ausencia cabeza ulnar del $\mathrm{m}$. pronador redondo 
lo pronador redondo, se encontró que en un $57 \%$ de los casos, el nervio mediano transitó entre las dos cabezas de origen (Figs. 1 y 2). En un $40 \%$ de los casos, el nervio mediano perforó las fibras pertenecientes a la cabeza ulnar del músculo pronador redondo (Figs. 1, 2 y 3).
Existió un único caso $(3,0 \%)$ en que no se presentó ninguna de las disposiciones descritas, debido a que la cabeza ulnar de este músculo estuvo ausente, por lo cual, en este caso el nervio mediano transitó posterior a la cabeza humeral de dicho músculo.

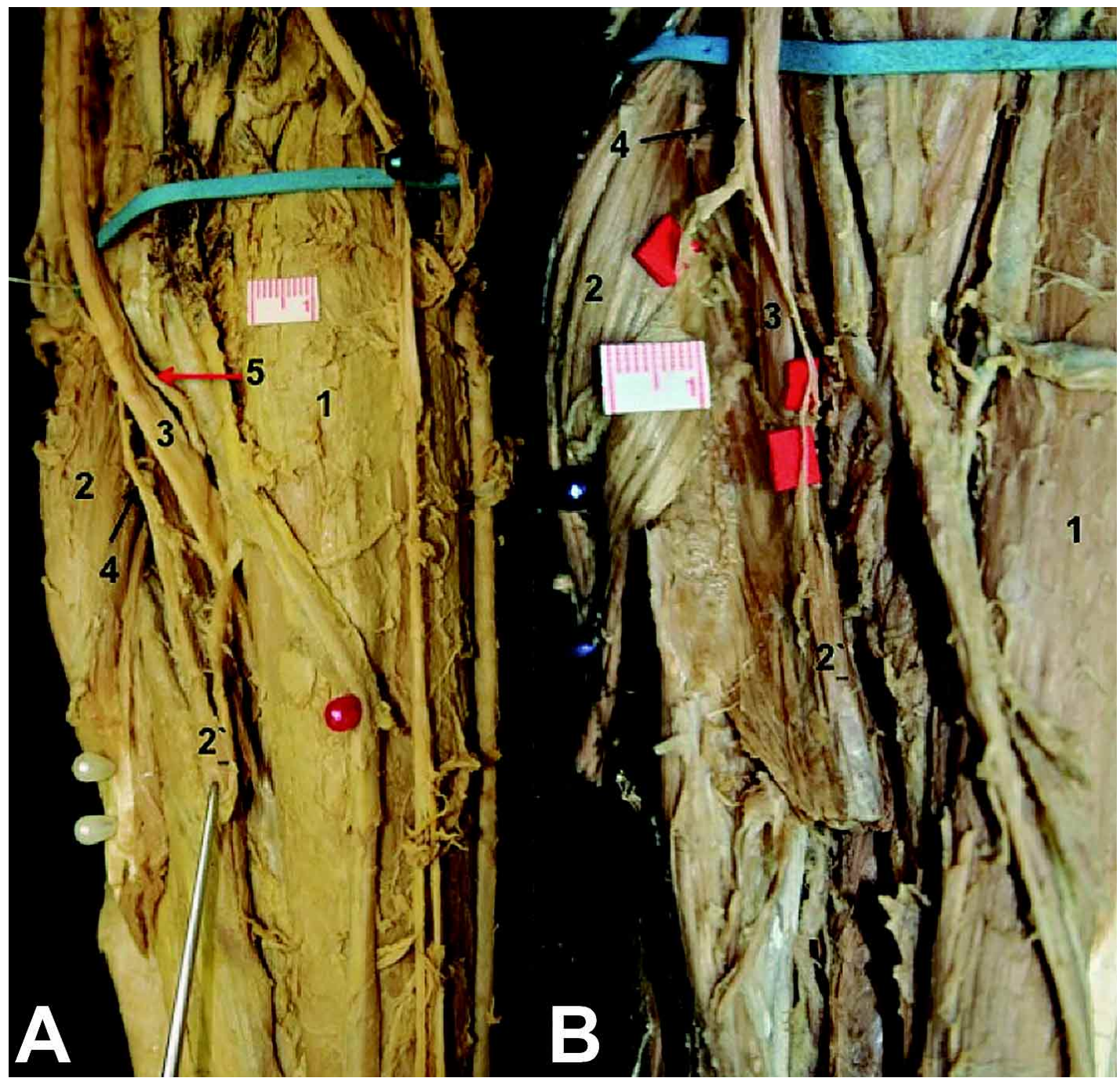

Fig. 3. 1= Músculo Braquiorradial; 2= Músculo Pronador Redondo, cabeza humeral; 2’= Músculo Pronador Redondo, cabeza ulnar; 3= Nervio mediano; 4= Tronco común de los motores para el músculo pronador redondo; 5= Tronco común para los ramos de los músculos palmar largo y flexor radial del carpo.

\section{DISCUSIÓN}

Al analizar la potencial compresión del nervio mediano en su paso a través del espacio existente entre las cabezas del músculo PR a nivel de la fosa cubital, se puede señalar que en este punto la gran mayoría de los autores como Tillaux (1880), Gérard, Testut \& Latarjet, Khale et al. (1994), Williams et al., Moore \& Dalley (2002) y
Rouvière \& Delmas, señalaron que el nervio mediano atraviesa este músculo entre sus cabezas humeral y ulnar. Sin embargo, los autores Sappey, Poirier \& Charpy (1904), Prives et al. y Tountas \& Bergman (1993), plantearon la posibilidad de que el nervio mediano se disponga posterior a la cabeza ulnar o perfore el vientre muscular de esta 
cabeza de origen del músculo pronador redondo. En el presente estudio se observó que el nervio mediano transitó entre las dos cabezas de origen del músculo PR en el $57 \%$ de los casos, diferente de la presentada por Beaton \& Anson, quienes en una serie de 240 miembros superiores, encontraron que en un $82,5 \%$ de los casos, este nervio mediano se ubicó entre la cabeza ulnar y humeral del pronador redondo.

En relación a las implicancias clínicas de estos fenómenos es importante señalar el concepto expresado por Nakano (1978), quien establece que este atrapamiento se describe conceptualmente como la irritación mecánica por la cual un nervio especifico se lesiona locamente, en un sitio anatómico vulnerable.

Uno de los trastornos generados por estos atrapamientos es conocido como síndrome del pronador el cual puede ser provocado por fractura, luxaciones, tumores en la región de la articulación del codo, o por la presencia de estructuras que generen una compresión extrínseca del nervio, entre las que destaca, la ya descrita, zonas de paso entre las cabezas del músculo PR, la hipertrofia del mismo, la presencia del ligamento de Struthers, el paso por la arcada del músculo flexor superficial de los dedos, el excesivo espesor del lacertus fibroso, la presencia de aneurismas, trombosis venosas o trayectos vasculares anómalos (Johnson et al.,1979; Nigst \& Dick; Fuss \& Wurzl, 1990; Braun \& Spinner, 1991; Olehnik et al., 1994; Williams et al.; Moore \& Dalley; Schünke et al., 2005; Pró, 2012).

Entre los hallazgos clínicos de esta compresión a nivel de la fosa cubital destaca la debilidad de todos los músculos inervados por el nervio mediano en el antebrazo y mano. A esto se suma parestesias y dolor difuso a nivel de la mano. Por otra parte, la pronación resistida del antebrazo con la mano en flexión también produce dolor. El tratamiento inicialmente comprende reposo y si no se produce mejoría, descompresión quirúrgica (Olehnik et al.; Pró).

Siendo más detallado en la descripción semiológica de este síndrome del pronador, Nigst \& Dick y Moore \& Dalley, describieron que entre los signos más importantes está la desaparición de la flexión de las articulaciones interfalángicas proximales del segundo y tercer dedo, a lo que se agrega el hecho de que se anula la flexión de las articulaciones interfalángicas distales del segundo y tercer dedos. Si bien no se afecta la flexión de las articulaciones distales del cuarto y quinto dedos debido a que la porción medial del flexor profundo de los dedos es inervada por el nervio ulnar, la capacidad de flexionar las articula- ciones metacarpofalángicas del segundo y tercer dedos se altera, ya que los ramos digitales del nervio mediano que inervan el primer y segundo lumbricales se ven comprometidos. Debido a esto, cuando el paciente trata de cerrar el puño, el segundo y tercer dedos quedan parcialmente extendidos, situación clínica conocida como "mano de predicador". A este hallazgo se le agrega la posibilidad de la aparición del signo de Tinel en la zona de inserción del músculo PR y del signo de la botella, en la cual, por la debilidad del músculo abductor corto del pulgar, la mano se ve impedida de realizar el cierre de la tapa de una botella.

A estos signos, Snell (2006) agrega la debilidad de la flexión de muñeca, la que se acompaña con una desviación ulnar de la mano. Esta última desviación es causada por la parálisis del flexor radial del carpo, que sumada a la intacta fuerza del flexor ulnar del carpo y la mitad medial del flexor profundo de los dedos, produce un desbalance muscular que genera la dicha desviación. En la zona de la mano, los músculos de la eminencia tenar se paralizan y se atrofian por lo que se aplana la región palmar lateral, lo que se suma a la ausencia de la oposición del pulgar y una severa complicación para realizar la función de prensión, situación que también se presenta en el síndrome del canal carpiano. Respecto al compromiso sensitivo, debemos destacar que la somatoestesia de la piel se pierde en la mitad lateral de la palma de la mano, la cara palmar y la parte distal de las superficies dorsales de los tres dedos laterales, sumado a la mitad lateral del dedo anular.

Buscando detalles respecto a este síndrome, debemos destacar que el nervio interóseo anterior generalmente nace del nervio mediano por encima del punto de compresión. Sin embargo, puede verse afectado en conjunto con el nervio mediano o por sí solo. Si se trata de diferenciar estos dos cuadros de compresión nerviosa debemos mencionar que cuando el paciente sufre compresión del nervio interóseo anterior se genera una debilidad de la pinza digital por afección de los músculos flexor largo del pulgar y el vientre del flexor profundo de los dedos que se dirige hacia el dedo índice. Una de los signos clínicos que demuestra esta debilidad está caracterizado por la incapacidad de realizar una letra "O" entre los dedos índice y el pulgar. Uno de los elementos que puede ocasionar que esta forma de presentación no sea tan clara, radica en la posibilidad de que el paciente presente ramos comunicantes entre los nervios interóseo anterior con el nervio ulnar (ramo comunicante de Martin-Gruber) (Nakano; Nigst \& Dick; Williams et al.; Leversedge et al., 2010).

Otra de las diferencias que se observó en este estudio respecto a lo expresado por Beaton, \& Anson se vin- 
culó a que en el $40 \%$ de los casos estudiados, el nervio mediano perforó las fibras pertenecientes a la cabeza ulnar del músculo pronador redondo, trayecto que los autores no describen entre sus hallazgos. A esta diferencia se suma a que en el $8,75 \%$ de sus casos, la cabeza ulnar de este músculo estuvo ausente, situación que en nuestra serie sólo alcanzó el 3\% de la muestra. Por otra parte, nuestro estudio también difiere con los resultados de von Lanz \& Wachsmuth, que describieron que en el 95\% de los casos, el nervio discurre entre las dos cabezas del músculo pronador redondo, dejando a un $2 \%$ de los casos, como aquellos en la cual el nervio mediano perforó la cabeza humeral del músculo pronador redondo y en el $3 \%$ restante el nervio mediano se dispuso por debajo de la cabeza ulnar.

Si nos basamos en la posibilidad de que el nervio mediano atraviese el vientre muscular ulnar del músculo $\mathrm{PR}$, se deduce una potencial compresión de este nervio dentro de la descrita cabeza muscular, lo cual, puede llevar a una serie de implicancias clínicas asociadas a sintomatología de los procesos de compresión nerviosa.

Para Nakano, esta entidad anátomo-clínica se describe conceptualmente como la irritación mecánica por la cual un nervio especifico se lesiona locamente, en un sitio anatómico vulnerable. Si este fenómeno se asocia a las profesiones u oficios que requieren el uso protagónico del miembro superior (temporeras, manipuladores de alimentos, etc), resulta importante establecer la correcta vinculación entre el sitio de compresión del nervio y la distribución de ramos que este nervio entrega proximal y distal al punto analizado. En esta línea, Fuss \& Wurzl, señalaron que el éxito de los tratamientos de descompresión están supeditados al conocimiento preciso y detallado de la distribución, número de ramos, punto de origen y punto de penetración de los mismos, respecto de los músculos inervados por dichos ramos.

$\mathrm{Al}$ respecto, Johnson et al., estudiaron 71 casos de descompresión del nervio mediano en la región proximal del antebrazo, de los cuales, 51 fueron sometidos a descompresión quirúrgica. Dentro de las observaciones clínicas que condujeron a estos pacientes a un procedimiento quirúrgico, se destaca la presencia de parestesias en los dedos pulgar, índice, medio y la mitad lateral del dedo anular, síntomas que se exacerbaban cuando se realizaba la flexión de codo y la pronación del antebrazo. Otro de los elementos clínicos llamativos fue la aparición de dolor en el antebrazo, síntoma que aumentaba cuando la pronación del antebrazo se realizaba en contra de una resistencia. Desde el punto de vista muscular, destacó la presencia de debilidad en la prensión junto con la aparición de signo de Tinel en la zona de inserción distal del músculo pronador redondo. Respecto a los resultados del procedimiento operatorio de los 51 pacientes seleccionados, en el $76 \%$ de los casos, el causante de la compresión fue la presencia de un arco fibroso entre las cabezas de origen del músculo PR. De los casos restantes, el 14\% sufrió atrapamiento por un arco fibroso que se formó entre las cabezas de origen del músculo flexor superficial de los dedos, dejando en un $4 \%$ de los casos, a los que la compresión se vinculó a un espesor excesivo del arco fibroso. En 47 de los 51 de los casos, los síntomas desaparecieron posterior a la cirugía, en un promedio de 9 meses.

Otra perspectiva la entrega la investigación de Olehnik et al., que analizaron los resultados del tratamiento operatorio de descompresión del nervio mediano en correlación con el lugar de su atrapamiento. Para ello se examinaron 39 miembros superiores, de los cuales, en 34 se presentaba dolor del antebrazo y en 19 se sumó dolor en mano y/o muñeca. A esto se agregó que en 21 pacientes se presentó debilidad en la prensión. En todos los pacientes se aplicó el mismo procedimiento operatorio, en donde se analizó cada uno de los potenciales sitios de compresión, encontrándose en 22 casos una compresión del nervio mediano por parte de la arcada fibrosa del músculo flexor superficial de los dedos. En 13 de los casos se presentó en el trayecto que el nervio presenta entre las cabezas del músculo pronador redondo y en 4 de los pacientes se describió en ambos sitios. Es importante destacar que en 19 pacientes existió un procedimiento operatorio previo a nivel del túnel del carpo. Dentro de los resultados obtenidos en el estudio se observó mejoría completa en 10 de los pacientes, 20 de los restantes aliviaron sus síntomas, quedando 9 sin mejoría. De los 19 pacientes operados previamente en el túnel del carpo, 14 mejoraron su condición. De los 22 con compresión en la arcada fibrosa del músculo flexor superficial de los dedos, 18 mejoraron y lo mismo sucedió en 9 de los 13 pacientes con compresión por parte del músculo PR.

Todo este análisis representa una evidencia importante al momento de considerar el correcto abordaje de las cirugías de liberación del nervio mediano, que en su mayoría se centran en liberar el nervio mediano en su trayecto dentro del canal del carpo. En esta misma línea, Olave et al. (2002) señalaron que el conocimiento de la inervación de los músculos y de sus variaciones es de gran importancia para realizar el diagnóstico correcto cuando algún nervio ha sido lesionado y sin duda también, para realizar un adecuado tratamiento, agregando que la mayoría de los textos de anatomía describen bien los trayectos, pero no detallan sus ramos de distribución, el número de ramos secundarios y menos sus lugares de ingreso en el vientre muscular. 
RIVEROS, A.; OLAVE, E. \& SOUSA-RODRIGUES, C. Relationships between median nerve and the pronator teres muscle in the cubital region: anatomic and clinical implications. Int. J. Morphol., 33(4):1448-1454, 2015.

SUMMARY: Intense muscle activity of the forearm generates the need to describe the possible sites of compression of the peripheral nerves of the upper limb. For the median nerve one of the most prevalent sites of nerve entrapment is when the nerve travels within the carpal tunnel. To this we must add the potential compression of the nerve when it passes through the heads of the pronator teres muscle (PT). This compression is characterized by paresis of the wrist flexor muscles or some of the thenar, signs which can add to numbness and pain in the hand. The aim of this study was to describe the relation of the median nerve with the PT muscle. Thirty formolized forearms of adult Brazilian individuals were used. It was found that in $40 \%$ of cases the median nerve crossed the ulnar head PT muscle. In one case the ulnar head was absent. Due to this, the median nerve passed posterior to the humeral head of this muscle. The clinic that develops from compression syndrome at this level is directly linked to the distribution of the branches that the nerve presents proximal to the site of compression. Associated with these findings, it is vital for optimal diagnosis of the compression of median nerve to rule out compression of this nerve in proximal sites to the carpal tunnel.

KEY WORDS : Anatomy; Median nerve; Pronator teres muscle; Compression syndrome.

\section{REFERENCIAS BIBLIOGRÁFICAS}

Beaton, L. E. \& Anson, B. J. The relation of the median nerve to the pronator teres muscle. Anat. Rec., 75(1):23-6, 1939.

Braun, R. M. \& Spinner, R. J. Spontaneous bilateral median nerve compressions in the distal arm. J. Hand. Surg. Am., 16(2):2447, 1991.

Fuss, F. K. \& Wurzl, G. H. Median nerve entrapment. Pronator teres syndrome. Surgical anatomy and correlation with symptom patterns. Surg. Radiol. Anat., 12(4):267-71, 1990.

Gérard, G. Manuel d'anatomie humaine. 2a ed. Paris, Masson, 1912.

Gross, P. T. \& Jones, H. R. Jr. Proximal median neuropathies: electromyographic and clinical correlation. Muscle Nerve, 15(3):390-5, 1992.

Johnson, R. K.; Spinner, M. \& Shrewsbury, M. M. Median nerve entrapment syndrome in the proximal forearm. J. Hand Surg. Am., 4(1):48-51, 1979.

Khale, W.; Leonhardt, H. \& Platzer, W. Atlas de Anatomía. Vol. 3. 5a. ed. Barcelona, Omega, 1994. p.375.

Leversedge, F. J.; Goldfarb, C. A. \& Boyer, M. I. A pocketbook Manual of hand and upper extremity anatomy: Primus manus. Philadelphia, Lippincott Williams \& Wilkins, 2010. p.256.

Moore, K. \& Dalley, A. Anatomía con orientación clínica. 4a ed. Buenos Aires, Médica Panamericana, 2002. p.1185.

Nakano, K. K. The entrapment neuropathies. Muscle Nerve, 1(4):264-79, 1978.

Nigst, H. \& Dick, W. Syndromes of compression of the median nerve in the proximal forearm (pronator teres syndrome; anterior interosseous nerve syndrome). Arch. Orthop. Trauma Surg., 93(4):307-12, 1979.
Olave, E.; Gabrielli, C.; Braga, M. T. T. \& del Sol, M. Aspectos biométricos de los ramos motores del nervio musculocutáneo para el músculo braquial. Rev. Chil. Anat., 20(2):231-6, 2002.

Olehnik, W. K.; Manske, P. R. \& Szerzinski, J. Median nerve compression in the proximal forearm. J. Hand Surg., 19(1):1216, 1994.

Poirier, P. \& Charpy, A. Traité d'anatomie humaine. 2a ed. Paris, Masson et Cie, 1902.

Prives, M.; Lisenkov, N. \& Bushkovich, V. Anatomía humana. Vol. 3. 5a. ed. Moscú, MIR, 1984. p.484.

Pró, E. Anatomía Clínica. Buenos Aires, Médica Panamericana. 2012. p.992.

Rauber, A. Anatomie des Menschen. Vol. 2. 5a ed. Leipzig, Verlag von Arthur Georgi, 1898. pp.882.

Rouvière, H. \& Delmas, A. Anatomía humana: descriptiva, topográfica y funcional. Vol. 3. 11a ed. Barcelona, Masson, 2005.

Sappey, P. C. Traíte D`Anatomie Descriptive. Vol 3. 4a. ed. Paris, Lecrosnier et Babé, 1889.

Schünke, M.; Schulte, E. \& Schumacher, U. Prometheus: Texto y Atlas de Anatomia. Buenos Aires, Médica Panamericana, 2005.

Sinelnikov, R. Atlas de anatomía humana. Vol. 3. 4a. ed. Moscú, MIR, 1986. p.397.

Snell, R. Clinical Anatomy for Medical Students. 6a. ed. Philadelphia, Lippincott Williams \& Wilkins, 2006. p.960.

Testut, L. \& Latarjet, A. Tratado de Anatomía Humana. Vol. 3. 9a ed. Barcelona, Salvat, 1972. 
RIVEROS, A.; OLAVE, E. \& SOUSA-RODRIGUES, C. Relaciones entre nervio mediano y musculo pronador redondo en la región cubital: implicancias anatomo-clínicas. Int. J. Morphol., 33(4):1448-1454, 2015.

Tillaux, P. Tratado de Anatomía Topográfica: Aplicada a la Cirugía. 12a . ed. Barcelona, Hijos de J. Espasa, 1880.

Tountas, C. \& Bergman, R. Anatomic variations of the upper extremity. New York, Churchill Livingstone, 1993. p.286.

Tubbs, R. S.; Jones, V. L.; Loukas, M.; Cömert, A.; Shoja, M. M.; Wellons, J. C. 3rd. \& Cohen-Gadol, A. A. Anatomy and landmarks for branches of the brachial plexus: a vade mecum. Surg. Radiol. Anat., 32(3):261-70, 2010.

von Lanz, T. \& Wachsmuth, W. Praktische Anatomie, 2 Aufl. Bd 1/3: Arm. Berlin, Springer, 1959. p.144.

Williams, P. L.; Bannister, L. H.; Berry, M. M.; Collins, P.; Dyson, M.; Dussek, J. E. \& Ferguson, M. W. J. Anatomía de Gray. Bases Anatómicas de la Medicina y la Cirugía. $38^{\mathrm{a}}$ ed. Madrid, Churchill Livingstone-Harcourt Brace, 1998.

\section{Dirección para Correspondencia Mg. Andrés Riveros \\ Depto. de Ciencias Morfológicas \\ Universidad San Sebastián \\ Sede Concepción \\ CHILE}

Email: ariverosmed@gmail.com

Recibido : 12-07-2015

Aceptado: 22-09-2015 